\title{
Retos, perspectivas y consideraciones éticas en torno a la evaluación del lenguaje en la persona con demencia
}

\author{
Challenges, perspectives and ethics considerations over the language \\ assessment of the person with dementia
}

\author{
Beatriz Valles González ${ }^{1}$
}

\begin{abstract}
RESUMEN
El presente ensayo plantea una reflexión en torno a los retos, perspectivas y dilemas éticos que se presentan al evaluar el lenguaje de las personas con demencia. Esta se hizo desde dos perspectivas, una de tipo epistemológica-ontológica, dirigida a estudiar el concepto de trastorno del lenguaje y su relación con el envejecimiento. La otra, metodológica, relacionada con el análisis de las técnicas de evaluación lingüística utilizadas en el diagnóstico de las alteraciones lingüísticas de esta población. A partir de ella se proponen diferentes alternativas como: revisar el concepto de trastorno, redefinir el objetivo de la evaluación del lenguaje y profundizar en el conocimiento del funcionamiento lingüístico del adulto mayor sano como paso previo para el uso del análisis del discurso como herramienta válida para valorar la eficiencia lingüística de las personas que padecen demencia. Todas estas acciones pueden garantizar una atención ética y ajustada a las necesidades lingüísticas reales de este colectivo y de su familia.
\end{abstract}

Descriptores: Enfermedad de Alzheimer; Evaluación/métodos; Lenguaje; Bioética

\section{INTRODUCCIÓN}

El interés por las demencias en el ámbito clínico ha aumentado en las últimas décadas, motivado a las variaciones demográficas que se están presentando tanto en países desarrollados y como en aquellos en proceso de desarrollo, las cuales han elevado la esperanza de vida por encima de los ochenta años de edad. En el año 2000 existían en el mundo 600 millones de personas mayores de 60 años, para los próximos 20 años se espera que esta cifra aumente en un $70 \%{ }^{(1)}$. El rápido incremento en la población de ancianos trae un aumento de los casos de problemas físicos y mentales relacionados con la edad, y además, un mayor riesgo de demencia para este colectivo ${ }^{(2)}$.

Esta situación por una parte, posibilita el incremento de los casos de demencia y ha provocado por lo tanto, una verdadera alarma de salud pública en el mundo desarrollado, obligando

Esta investigación fue posible gracias al apoyo financiero del Fogarty Internacional Collaborative Research Training Grant, de la Universidad de Chicago y de La Universidad del Zulia.

(1) Doctora, Fellow Posdoctoral en Bioética en el MacLean Center (The University of Chicago) - Chicago (IL), Estados Unidos; Profesora Asociada del Departamento de Educación Especial del Instituto Pedagógico de Caracas de la Universidad Pedagógica Experimental Libertador - Caracas (Distrito Capital), Venezuela.

Endereço para correspondência: Beatriz Valles González. Av. Andrés Be1lo, Calle Buenos Aires, Res. Belloral PH 125, Los Caobos, Caracas 1050, Venezuela. E-mail: brvallesg@gmail.com

Recebido em: 13/12/2006; Aceito em: 27/8/2007 al diseño de nuevas políticas sanitarias dirigidas a la atención integral de las personas por encima de los 65 años $^{(3)}$. Pero por otra, ha dejado ver la necesidad de planificar la atención del adulto mayor tomando en cuenta algunos principios éticos para lograr una actuación apegada a lo que se denomina una ética gerontológica adecuada al momento presente ${ }^{(4)}$, la cual demanda de parte de los equipos de interventores una nueva geometría de atención que obliga a profundizar en los aspectos sociales y en las demandas de los contextos de vida de los usuarios de los servicios de salud.

El propósito del presente trabajo es reflexionar en torno a las posibilidades actuales de la evaluación lingüística y en los retos, perspectivas y dilemas éticos que se presentan al desarrollar esta actividad con la persona con demencia (en adelante PCD). Esta reflexión se hizo desde dos perspectivas. Una de tipo epistemológica-ontológica, dirigida a estudiar el concepto de trastorno del lenguaje y su relación con el proceso de envejecimiento. La otra, metodológica, relacionada con el análisis de las técnicas de evaluación lingüística utilizadas en el diagnóstico de la población de adultos mayores. A partir de ella, se propone una revisión del concepto de trastorno del lenguaje, una mayor investigación sobre el funcionamiento lingüístico del adulto mayor sano con base al estudio de sus habilidades discursivas y la revisión de las herramientas diagnósticas utilizadas en el área, como pasos previos para el diseño de propuestas complementarias basadas en el uso del análisis del discurso como un recurso necesario para valorar 
la eficiencia lingüística en las PCD y en la planificación de la atención de los trastornos del lenguaje en esta población.

\section{REVISIÓN DE LITERATURA}

\section{Lo epistemológico y lo ontológico como elementos bási- cos en la definición del trastorno del lenguaje}

Las PCD exhiben diferentes manifestaciones, algunas a nivel orgánico, llamadas marcadores biológicos, que pueden ser detectadas con pruebas especiales, como por ejemplo cambios en el volumen del hipocampo, la presencia de la apolipoproteina E4 o de las placas amiloideas en los casos de la demencia tipo Alzheimer. Otros síntomas son las fallas en la memoria y en la función ejecutiva, la depresión, los trastornos del lenguaje y las dificultades en la atención ${ }^{(5)}$. Este último grupo de manifestaciones puede ser llamado los marcadores psicosociales, pues si bien responden al proceso de deterioro cognoscitivo típico del síndrome demencial, están influenciadas por factores sociales como: el estilo de vida, el nivel de instrucción, el tipo de dieta y la actividad física.

De todos los marcadores psicosociales, el concepto de trastorno del lenguaje es el más borroso y confuso, pues remite a diferentes cuadros diagnósticos que intentan definir y aclarar el tema, pero muy lejos de esto terminan siendo una suerte de etiquetas diagnósticas que no explican plenamente la conducta lingüística del sujeto evaluado. El motivo de esta indefinición puede estar en lo difícil que resulta precisar a qué llamamos rendimiento normal cuando nos referimos a la conducta lingüística del adulto mayor. Desde la ventana médica, toda Nosografía o todo intento de clasificar las enfermedades requiere un paso previo: una teoría del enfermar. Nos gustaría utilizar esta propuesta para poder aclarar cómo se manifiesta un trastorno del lenguaje y en consecuencia, a qué remite este concepto $^{(6)}$.

El trastorno del lenguaje se define en la clínica fonoaudiológica, como una actuación lingüística fuera de la norma. Es decir, cualquier desviación en los componentes de la lengua (fonológico, semántico, morfológico y sintáctico), producto de un desorden orgánico o funcional que no se corresponda con ese "hablante ideal" usuario de la norma culta o estándar. El problema al definir lo trastornado en la actuación lingüística o hablar de norma adecuada o inadecuada, es que éste es un tópico que precisa un enfoque integrador más allá de lo lingüístico. A partir de esta propuesta, se debe asumir el lenguaje y sus alteraciones, no sólo desde lo neurológico, sino en términos de un proceso influenciado por variables sociales y culturales, las cuales juegan un rol importante en su definición y caracterización. Por ello, para estudiar el trastorno se hace necesaria una visión amplia que incluya lo lingüístico, lo psicológico y lo social y además, hacerlo desde un marco interdisciplinario y complejo, donde diferentes saberes y puntos de vista se conjuguen.

La norma lingüística es definida como el sistema de reglas que presenta lo que se puede elegir entre los usos de una lengua determinada, si se quiere estar de acuerdo con cierto ideal estético o sociocultural. El problema es que ese "ideal" es definido por un grupo que podemos llamar culto, pero que no necesariamente comparte lo que es considerado como norma por otros grupos. Por ello, la elección de lo normativo o de lo estándar se convierte en una relación de poder, pues este concepto remite a las creencias que un grupo tenga con respecto a cómo deben producirse sus actos de habla, a su esquema cognoscitivo o ideológico con respecto al uso del lenguaje y a cómo concretar el uso de su lengua en determinados contextos. De allí que se relacione con lo sociolingüístico.

Definir norma y trastorno nos conduce a la consideración de variables epistemológicas y ontológicas, es decir a teorías y a creencias. En otras palabras, a nuestros marcos ideológicos y a nuestro mundo posible. Con respecto a este punto, cabe destacar que el concepto de estándar es más una comodidad teórica que una realidad ${ }^{(7)}$, pues al usar cualquier lengua, los hablantes manifiestamos una gran variedad de formas, todas ellas altamente productivas desde lo discursivo, y esta variabilidad no se relaciona sólo con el paso de los años o con el nivel de instrucción, sino que la misma es una realidad sociolingüística que debe ser considerada al diseñar instrumentos de evaluación.

Por ello se debe profundizar en este aspecto, pues el mismo explica porqué la interacción con el anciano está marcada por creencias que hacen posible que la conversación entre jóvenes $\mathrm{y}$ viejos sea un intercambio influenciado por ideas como: el anciano no entiende, no puede hablar de determinados tópicos, no sigue la conversación, no comprende....En fin, un conjunto de creencias o estereotipos basados en mitos acerca de la vejez que llevan a los más jóvenes a creer que el anciano tiene una ejecución lingüística diferente o una norma distinta ${ }^{(8)}$.

La consideración negativa que se tiene de la actuación linguística del adulto mayor, marcada por la idea del envejecimiento como un sinónimo de decrepitud o pérdida de facultades, tan común en una sociedad que tiende a alabar lo perfecto, ha permitido que lo relacionado con la investigación de la actuación lingüística del anciano se asuma en gran parte como el estudio de lo patológico. Si el investigador asume al anciano como portador de trastornos, su proceso de investigación estará condicionado a la búsqueda de estos y por lo tanto la descripción de las variaciones normales en esta etapa de la vida puede ser abordada de manera inadecuada, al no tener en cuenta los distintos factores que convergen en esta etapa del desarrollo humano ${ }^{(9)}$.

La actividad comunicativa y lingüística de los mayores está marcada por procesos paralelos, unos de tipo social, como puede ser el pasar a quedarse solo en su hogar luego de la mudanza de sus hijos o el cambio en su desempeño como sujeto productor a partir de la jubilación o del retiro de su trabajo, ambos eventos impactan de forma negativa pues reduce las posibilidades de interacción social. Otros factores de carácter biológico, provocan una serie de alteraciones tanto en la estructura física (músculos, huesos) como en la función de diversos sistemas: cardiovascular, respiratorio, endocrino, auditivo y visual. Dentro de todos estos cambios, el cognoscitivo es quizás el que provoca los efectos más negativos, pues determina una reducción en la velocidad de respuesta y limita la capacidad de procesamiento. Sin embargo, la mayoría adultos mayores que presentan un envejecimiento normal pueden exhibir una adecuada ejecución comunicativa ${ }^{(10)}$. 
Como parte de un proceso de envejecimiento normal se produce un deterioro de la memoria operativa, éste limita el sistema atencional y de control, especialmente los procesos de atención y de inhibición que implican selección, planificación y supervisión. Todo ello afecta los aspectos lingüísticos más relacionados con la memoria, como: el acceso al léxico, la comprensión y producción de oraciones complejas y la comprensión y producción del discurso. Estos cambios deben ser analizados teniéndolos como típicos para este momento del desarrollo humano y aceptando que en el anciano el tiempo para procesar la información aumenta, pero esta variación no determina la imposibilidad para actuar de manera exitosa en lo lingüístico. De allí que el diagnóstico en el área del lenguaje debe considerar todos estos datos y relacionarlos con los aspectos sociales y culturales en torno al proceso de envejecimiento ya señalados.

Lo anterior obliga a los evaluadores a estudiar el proceso de envejecimiento sano en una perspectiva integral, la cual aporte datos fieles, que permitan explicar y caracterizar las variaciones típicas de la interacción lingüística que se dan en el anciano sano, para a partir de ellos, poder comprender el deterioro que producen la demencia u otros cuadros como el trastorno cognoscitivo leve. Esto supone una limitación en la evaluación, que necesita ser atendida con celeridad, pues representa el establecer un posible diagnóstico de lo patológico, sin contar con una adecuada definición de lo normal en algunos países con una población mayoritariamente joven, en los cuales no existen suficientes datos sobre el proceso de envejecimiento típico de sus habitantes, como es el caso de Venezuela; además, requiere que el evaluador tome en cuenta que los seres humanos respondemos desde nuestra experiencia de vida, por lo que no existen soluciones únicas a los problemas, sino más bien una gama de posibles conductas aceptables en respuesta a una tarea dada o a una pregunta ${ }^{(11)}$.

Volviendo al concepto de trastorno del lenguaje, podemos afirmar que en una perspectiva general, debe ser asumido como la manifestación de una alteración de base orgánica, cognoscitiva, motora o neurológica. Su presencia produce la reducción en la competencia linguística y comunicativa de acuerdo con la norma, lo que se evidencia por una parte como una limitación en la capacidad de producir textos orales y/o escritos con adecuada coherencia y cohesión, y por otra, en la imposibilidad de comprenderlos.

De esta manera, el trastorno siempre será la consecuencia de una alteración orgánica o psicológica e incluye las alteraciones en el uso de la lengua oral y de la escrita. Pero en este punto es necesario el uso de las taxonomías, pues si bien estas intentan aclarar las características de los diferentes cuadros patológicos, al tener en cuenta las descripciones cualitativas de los síntomas de los pacientes surgen problemas para sustentar esta clasificación clínica. Un ejemplo de esto es lo que sucede al evaluar a personas con afasias o en el estudio de los trastornos del lenguaje asociados con las demencias, pues si bien podemos establecer diferencias en los síntomas lingüísticos de acuerdo con los diversos tipos, los sujetos con afasia y las PCD (como cualquier hablante) pueden manifestar variaciones relacionadas con lo dialectal, con su nivel de instrucción, o con factores emocionales, razones que dificultan el ubicar la conducta lingüística dentro de determinado patrón.
Además, se debe tener en cuenta las variaciones en la ejecución lingüística entre los individuos que conforman ese gran grupo denominado como adultos mayores, pues entre el viejo-viejo (80 años) y el viejo-joven (70 años) existen diferencias de ejecución cognoscitiva y lingüística que deben ser consideradas a la luz de diversas variables como salud, escolaridad y calidad de sus relaciones sociales ${ }^{(12)}$. De allí, que todo evaluador debe conocer la historia de vida del sujeto que se está evaluando, manejar las características sociolingüísticas de la lengua, entender cómo la conversación se ve influenciada por una serie de costumbres y creencias que afectan este intercambio con los adultos mayores y analizar cómo el trastorno afecta la calidad de vida del sujeto que es evaluado.

Actualmente es imprescindible investigar en un marco de colaboración interdisciplinaria, donde lingüistas, psiconeurólogos, médicos y fonoaudiólogos, trabajen en torno al beneficio de reconducir la descripción y conceptualización de este término. Para esto, es necesario: 1) aceptar el elevado nivel de incertidumbre que existe en el estudio de los trastornos del lenguaje y especialmente en lo relacionado con la atención de aquellos de base neurológica; y 2) trabajar mas en función de la descripción y explicación del desempeño del sujeto evaluado.

Esta transformación que está contenida en la Clasificación $\mathrm{CIF}^{(13)}$, la cual fue por elaborada por la Organización Mundial de la Salud a partir de un enfoque biopsicosocial, exige por parte de los profesionales involucrados el reconocimiento de la necesidad de cambio en el paradigma de evaluación, hecho que requiere de un profundo análisis epistemológico y de una transformación metodológica mediada por la lógica revisión ontológica del equipo evaluador. No se trata de dejar de lado la búsqueda de los correlatos lesión y alteración de la función, o de no diferenciar los síntomas de los diferentes tipos de demencias, elementos vitales en la atención del paciente, sino más bien esforzarnos en integrar al proceso evaluativo suficientes datos sobre las características del discurso de la PCD, atendiendo en este análisis cualitativo categorías procedentes de lo textual y de lo contextual. En otras palabras, se insiste en asumir el lenguaje como un fenómeno social, determinado por variables socioculturales que deben ser valoradas como un elemento esencial en la exploración de la calidad de la interacción lingüística.

\section{Trastornos del lenguaje y demencias}

El lenguaje de las PCD presenta una serie de características que han sido estudiadas por varios autores ${ }^{(14-17)}$, las cuales varían de un paciente a otro y de acuerdo con la progresión y etiología de la demencia. Las más resaltantes son la anomia, la parafasia, el uso de neologismos y de muletillas o palabras carentes de sentido, un ligero aumento en la longitud de las emisiones verbales y severas dificultades por seguir el tópico de una conversación. Todos estos trastornos provocan una comunicación difícil y costosa entre paciente e interlocutor. La dificultad aumenta cuando este último es un extraño al contexto diario y a la historia de vida de la PCD, como puede ser el fonoaudiólogo, las enfermeras o los médicos. 
El lenguaje de las PCD deja de ser normal y comienza a trastornarse de manera progresiva, en razón de los problemas que el sujeto manifiesta en el uso de una serie de recursos cohesivos como por ejemplo el empleo de las preposiciones, o por las limitaciones en establecer la concordancia de número o en lo relacionado con recordar o seleccionar la palabra correcta. Pero además, el discurso de la PCD puede mostrar limitaciones en cuanto a la coherencia resultado de una inadecuada lectura del contexto, por lo que no llega a ajustarse a los rápidos cambios temáticos y en consecuencia reducir sus turnos de habla o hablar sin atender el tema tratado.

Todas estas variables afectan la posibilidad de la PCD para actuar lingüísticamente de manera eficiente. Además, en todo este proceso es necesario considerar la actitud de su interlocutor, quien puede colaborar en la construcción del texto o por el contrario, mostrar su rechazo en ser parte de este contexto. El discurso de la PCD podría ser considerado como trastornado, en razón de las dificultades lingüísticas que en éste se manifiestan y por la necesidad que tiene su interlocutor de buscar nuevas estrategias para lograr comprender lo que ella dice.

De acuerdo con lo anterior, podemos concluir que la PCD presenta un trastorno del lenguaje ya que su rendimiento lingüístico lo aparta de la norma que utilizan los adultos mayores que tienen un proceso de envejecimiento sano. Sus limitaciones lingüísticas más resaltantes se observan en el nivel semántico y hacen su comunicación difícil, especialmente en la etapa media y avanzada de este síndrome. En este punto, es necesario destacar que dependiendo de la topografía de la lesión la demencia produce diferentes síntomas lingüísticos, sin embargo clasificarlos en un cuadro único donde los distintos síntomas aparezcan organizados de forma absoluta es difícil, pues lo lingüístico estará siempre relacionado: con la localización de la lesión, con el progreso de la demencia y con variables ambientales y psicológicas, como por ejemplo, la calidad de la interacción verbal del grupo familiar.

En las demencias corticales como la de tipo Alzheimer y la Frontotemporal el paciente presenta síntomas como afasia, agnosia y $\operatorname{apraxia}^{(18)}$, estas manifestaciones no se encuentran en las demencias subcorticales como por ejemplo en la de tipo Frontotemporal, donde la disfagia, la disartria, la pérdida del discurso y de la fluidez verbal, son las características más frecuentes $^{(19)}$. Por otra parte, en las de tipo vascular se observa una combinación de estos síntomas. Sin embargo, en toda persona con demencia puede hacerse evidente la dificultad para actuar de manera ajustada a los marcos de referencia que rigen la conversación como el contexto primario para el intercambio lingüístico.

La conversación es un proceso básico de intercambio social y de construcción lingüística entre dos o más personas; y tiene al menos dos objetivos. El primero bastante obvio, está dirigido a transmitir información o contenido. El segundo, más difícil de apreciar, lleva a los interlocutores a establecer una relación de acuerdo mutuo, con base a la cual se construye la comprensión. Por ello toda conversación exitosa comienza con un proceso de colaboración, en el cual todos los participantes trabajan juntos de manera que se cree la comprensión y el sentido ${ }^{(20)}$.

La PCD tiene problemas para poder participar con eficiencia en este intercambio, al tener dificultades para leer el contexto y ajustarse a los cambios, turnos, variaciones del tema y otros eventos lingüísticos. De esta manera y como ya expresamos anteriormente, el trastorno se evidencia en un hecho objetivo como lo es la reducción de la coherencia y las dificultades manifiestas en el uso de los recursos cohesivos por parte del sujeto que la presenta.

Todos estos problemas pueden ser compensados a través de diferentes estrategias, como la reducción de la velocidad al hablar, una articulación más enfática o con el uso de las reparaciones conversacionales o correcciones que hacen paciente e interlocutor, para poder construir de manera adecuada y cooperativa la coherencia del discurso durante la conversación. $\mathrm{El}$ interlocutor del individuo con demencia debe desplegar recursos especiales, y adaptarse a las limitaciones en el uso del lenguaje que el progreso de la misma acarrea al paciente. Por esto, la interacción termina siendo muy costosa y la construcción de la conversación dependerá de las creencias que el interlocutor posea con respecto a la demencia como "enfermedad", a su relación emocional con el paciente y a su propio deseo de mantener el intercambio comunicativo a pesar de estos impedimentos.

Cabe destacar que el problema de comunicación con la persona con demencia no sólo afecta a su familia o a su cuidador, las limitaciones en este intercambio también afectan su comunicación con los proveedores de salud, incluyendo a los médicos y pueden provocar una reducción de la eficiencia de la comunicación médico-paciente ${ }^{(21)}$. Por ello, se hace necesario estudiar la actividad de todos los actores, pues la ejecución comunicativa del paciente se ve influida por la de su interlocutor, quien puede ayudarlo a mantenerse dentro de la conversación o reducir su interacción, hasta eliminar esta posibilidad de intercambio ${ }^{(17)}$.

Cómo este proceso de colaboración se construye entre los diferentes interlocutores y la actividad lingüística de ellos es el principal aspecto a ser estudiado durante la evaluación del lenguaje. Para alcanzar este objetivo se hace necesario utilizar nuevas herramientas diagnósticas, como por ejemplo, el análisis del discurso. Pero primero, se hace imprescindible comprender al anciano desde una perspectiva integral, donde variables psico-sociales (atención familiar, calidad de sus relaciones sociales, manejo de la jubilación o cambios en su estilo de vida), económicas (ingresos que garanticen calidad de vida, acceso a programas de protección y de prevención en salud) y físicas (estado de salud general, historia clínica, presencia de enfermedades crónicas) sean justamente valoradas al momento de decidir sobre la existencia del trastorno del lenguaje.

Lo anterior se expresa de forma dramática en los países en desarrollo, pues en estas latitudes el adulto mayor confronta una triple carga marcada por una marea ascendente de trastornos no transmisibles y degenerativos asociados con el envejecimiento, reducción en los niveles de sistemas de apoyo familiar, especialmente en los grandes centros urbanos, y falta de sistemas adecuados de seguridad social ${ }^{(22)}$. Esta realidad puede conducir por una parte, a un limitado acceso a los programas de salud dirigidos a prevenir la demencia y por otra, a un posible subregistro de casos, especialmente en las mujeres, quienes tradicionalmente deben enfrentar una pesada carga en el cuidado de la familia y en la búsqueda del sustento econó- 
mico, por lo que su salud puede verse seriamente amenazada en lo físico y en lo emocional ${ }^{(23)}$.

En estos países el anciano está en mayor riesgo nutricional, al no tener acceso a una dieta ajustada a sus necesidades biológicas, factor que lleva a un probable aumento de casos de demencia al influir negativamente en las conexiones entre factores nutricionales y neurodegeneración ${ }^{(24)}$.

Por todo lo planteado, la atención de la población mayor y por ende lo relacionado con la evaluación lingüística en las PCD amerita una profunda revisión. No se trata de elaborar nuevos testes o de diseñar otras pruebas diagnóstica, sino de favorecer un cambio en la concepción del sujeto que esta siendo tratado, en los propósitos del equipo evaluador y en la definición de los objetivos de la intervención de esta población desde un paradigma biopsicosocial, donde las conexiones sociales y las reales necesidades en comunicación sean tomadas en cuenta para garantizar una mejor calidad de vida para el adulto mayor y su familia.

Reconocemos que los cambios propuestos precisan de una mayor investigación de impacto en costos y en las exigencias que para los proveedores de salud representan, pero es necesario insistir en que los mismos significan una aproximación necesaria a la realidad de vida de los adultos mayores, por lo que creemos que son necesarios. Desde luego que este objetivo sólo podrá ser alcanzado si los equipos interventores son formados en una concepción social de la salud, la cual defina nuevos caminos a transitar, que incluyan a la comunidad como un todo y tomen en cuenta los valores culturales y las creencias de los diferentes grupos, tendencias que definen el cómo se concibe el proceso de envejecimiento y el cómo se atiende al colectivo de adultos mayores.

\section{DISCUSIÓN}

La evaluación del sujeto con demencia es un proceso laborioso, complejo y costoso que se realiza a partir de una serie de estrategias que pueden ser resumidas en la siguiente forma:

- Entrevista con el paciente y su familia

- Examen médico general, pruebas de laboratorio y otras como tomografía, radiografías y evaluaciones sensoriales

- Despistaje de trastornos psiquiátricos primarios

- Evaluación de las capacidades funcionales

- Una evaluación neuropsicológica comprensiva, que puede ser iniciada con el despistaje del deterioro cognoscitivo utilizando el Mini Mental Test ${ }^{(25)}$, y posteriormente con una evaluación formal utilizando pruebas normalizadas como la Escala de Evaluación Psicogeriátrica ${ }^{(26)}$ o el Examen del Trastorno Mental en los Ancianos de Cambridge ${ }^{(27)}$, entre otras estrategias diagnósticas.

Estas herramientas valoran el funcionamiento cognoscitivo y la función lingüística, con base a la aplicación de subpruebas que miden la capacidad para evocar a corto y a largo plazo información visual y auditiva, la capacidad para nominar, narrar eventos o describir objetos o escenas, y la función ejecutiva que se manifiesta por el control necesario para ejecutar instrucciones complejas o completar tareas que involucren el manejo de diferentes criterios.
Actualmente de una evaluación centrada sólo en la ejecución lingüística de la PCD se ha pasado a una tendencia en valorar la forma como el contexto influye en la conversación, por eso diferentes autores ${ }^{(14-15,20,28)}$ proponen el uso del análisis del discurso aplicado al estudio de la conversación de la PCD. Esta alternativa ha provocado la necesidad de revisar la actividad de los fonoaudiólogos, la de los neuropsicólogos y la de los médicos, pues la dinámica tradicional de evaluación del lenguaje ha dejado claro que el proceso diagnóstico debe ir más allá del paciente y abordar a la familia y a la comunidad; pero además, ha dejado ver la necesidad de redefinir el concepto de trastorno y el propio objetivo de esta evaluación, donde el interés del lingüista y del fonoaudiólogo por realizar el mejor análisis de lo comunicativo, se ajuste con la preocupación del médico por el establecimiento del diagnóstico.

La evaluación basada en el análisis del discurso ha permitido estudiar la dinámica de la comunicación entre el paciente y sus interlocutores (familiares, cuidadores, médicos y enfermeras) en una dimensión diferente, sin embargo, es necesario recordar que el contexto del hospital o del consultorio no permite establecer un clima donde la conversación sirva como vía para estudiar el uso de numerosos recursos cohesivos o la coherencia del discurso en el sujeto evaluado, por lo que actualmente el uso del análisis discursivo aplicado al estudio de la conversación de la persona con demencia precisa de mayor investigación, si bien es necesario reconocer que es una herramienta que precisa de mayor tiempo en su aplicación y genera por lo tanto el consumo de mayor tiempo y del esfuerzo de valorar no sólo la conducta lingüística de la PCD sino además la de sus interlocutores, como por ejemplo la de sus familiares y cuidadores.

La situación planteada representa una paradoja, pues si definimos el lenguaje como un proceso que permite la elaboración de textos para el intercambio social, la evaluación debe responder a esta consideración y evaluar eficiencia comunicativa en diferentes contextos y con diversos actores; y no quedarse en la repetición, enumeración o memorización de palabras, o en responder a preguntas en un ambiente poco natural y por lo tanto ajeno al contexto natural de intercambio linguístico.... ¿Cómo hacer para poder compensar esta limitación?

Consideramos que una salida válida es utilizar el análisis discursivo como herramienta que permita estudiar la interacción lingüística del sujeto con demencia. Esta estrategia trae como consecuencia un cambio en el paradigma de la evaluación lingüística, pues de un interés en identificar el trastorno exclusivamente en el paciente, debemos orientar la acción hacia la caracterización y la explicación de la conducta lingüística de todos los actores: paciente, cuidador, familiares y por supuesto, la de los propios proveedores de salud.

Desde luego que esta alternativa puede ser considerada como poco viable en el contexto clínico, no necesariamente por sus alcances o por su utilidad real, sino más bien por el esfuerzo en realizar procedimientos diagnósticos de tipo cualitativo, diferentes a los utilizados en la dinámica clínica por los fonoaudiólogos y neuropsicólogos. En otras palabras, la evaluación del lenguaje remite en la actualidad a incorporar no sólo un marco teórico nuevo, sino a considerar diferentes medios para la recolección de datos y para su estudio. Ello 
implica aproximarse más a los aportes de la lingüística y específicamente al estudio de la conversación desde lo discursivo, considerándola como el contexto ideal de actuación de los seres humanos.

Como ejemplo de esta dinámica cabe destacar los recientes esfuerzos en analizar las habilidades comunicativas de las personas con afasia, con base al estudio del uso de marcadores discursivos, donde se ha buscado analizar la actividad de estos hablantes en función de poder mantener la cohesión y la coherencia discursiva $^{(29)}$. O la evaluación de la conversación de la persona con demencia tipo Alzheimer realizada a partir del estudio del uso de las reparaciones conversacionales, proceso que se refiere a los esfuerzos de los interlocutores para corregir y resolver problemas en la compresión o en la captación auditiva de un mensaje $\mathrm{e}^{(14)}$. Este es un proceso que se manifiesta de forma compleja y variada y pueden ser clasificadas en:

a) Auto-iniciadas por el hablante causante del error, en contraposición a hetero-iniciadas, iniciadas por el interlocutor que percibe el error;

b) Auto-reparadas cuando el error es realmente enmendado por el hablante causante del mismo, o hetero-reparación cuando es llevada a cabo por el interlocutor ${ }^{(30)}$.

Este diálogo entre una PCD y su cuidador, muestra este tipo de reparaciones. En este caso una auto-reparada auto-iniciada y además, una hetero-reparación hetero-iniciada:

Paciente: Quiero tomar, tomar..., quiero... quiero... Tengo hambre.

Cuidador: Quieres comer, a ver, ¿quieres un sanduche?

Paciente, (sonriendo): Si, si, quiero eso.

El término reparación indirecta es utilizado para señalar la actividad de interlocutor (sano) de la persona con demencia tipo Alzheimer, quien a menudo debe solicitar información para poder conocer las intenciones del paciente ${ }^{(15)}$. Esto lo logra a través de marcadores discursivos (um-hum, uh) o parafraseando lo que él cree que quiere decir el paciente, para poder chequear de esta manera, si está comprendiendo el mensaje.

Aquí presentamos un ejemplo:

"Interlocutor: Y tú te distraes

Paciente: $\mathrm{Si}$

Interlocutor: ¿Después tú pierdes que es lo que quieres decir?

Paciente: Si, pero um, yo puedo, uh, esperar un poco Interlocutor: Um-hum“ (15).

El uso de las reparaciones remiten a la norma lingüística, especialmente las auto-iniciadas y las auto-reparaciones y sirven como una señal de monitoreo cognoscitivo efectivo. En otras palabras, son una muestra de que el hablante es capaz de identificar una falla en su expresión o de exigir información para comprender a su interlocutor. Por otra parte, son una muestra del interés que un hablante tiene por aquello que dice el otro. Este dato que puede ser considerado como algo trivial, representa un elemento muy importante en el estudio de los trastornos del lenguaje en la PCD, especialmente en lo relativo a la pragmática, pues su interlocutor se ve obligado a pedir clarificaciones y correcciones con mayor frecuencia, pero esta actividad amerita una gran motivación especialmente en etapas avanzadas de la demencia, cuando los trastornos del lenguaje aumentan.
La reparación indirecta juega un importante rol en la conversación del paciente con demencia durante las entrevistas en el contexto clínico, especialmente en el proceso para obtener consentimiento informado ${ }^{(20)}$. Al utilizar este recurso el entrevistador puede monitorear si la PCD comprende el tema que se está tratando y revisar su propia comprensión con respecto a los deseos o argumentos del entrevistado.

Por otra parte, el interlocutor de la PCD tipo Alzheimer usa reparaciones indirectas y hetero-reparaciones, las primeras dirigidas a chequear el tema o tópico de la conversación, por lo que están más ligadas a la coherencia global del discurso y las segundas con el objetivo de completar frases y corregir la forma de la expresión oral, por lo que están relacionadas con lo cohesivo. La PCD utiliza auto-reparaciones en pocas ocasiones. A medida que la demencia se agrava, los interlocutores sanos prefieren utilizar otras estrategias y en oportunidades reducen sensiblemente sus interacciones ${ }^{(17)}$.

Lo anterior podría estar relacionado con los valores que sobre la demencia poseen el público en general; pues la conversación entre adultos mayores y personas jóvenes contienen estrategias discursivas que reflejan y refuerzan creencias y estereotipos relacionados con la edad y con el proceso de envejecer ${ }^{(8)}$. Esto obliga a investigar a profundidad las características de la conversación con estos pacientes desde una perspectiva discursiva, donde como ya se dijo anteriormente, la actividad de todos los interlocutores sea valorada; lo que nos lleva a reflexionar en torno a la utilidad de este tipo de análisis en el proceso de evaluación y diagnóstico de los trastornos del lenguaje asociados con los diferentes tipos de demencias y plantea un campo de investigación futura en el área de la evaluación lingüística en la población de adultos mayores.

\section{Implicaciones éticas en la evaluación del lenguaje en los pacientes con demencia}

Lo limitado que resulta evaluar la comunicación fuera de un contexto natural, trae como consecuencia una actividad diagnóstica que puede generar datos insuficientes, y en ocasiones poco apegados a la realidad, como los que podemos obtener en las evaluaciones basadas exclusivamente en pruebas estandarizadas. Por ello, se hace necesario abordar la evaluación lingüística en un marco amplio que incluya a la familia, amigos y cuidadores del paciente. Esto precisa el replantear el concepto de trastorno del lenguaje y utilizar nuevas estrategias de evaluación que lleven a desarrollar las acciones más allá del consultorio y abordar el contexto de vida familiar de los pacientes. Sin duda que estos acuerdos harán posible una actividad diagnóstica más justa y ética por parte del equipo evaluador.

Consideramos como recurso válido a ser incorporado durante la evaluación de las $\mathrm{PCD}$, la elaboración de una extensa entrevista inicial e incorporar, a esta, la conversación sobre temas de interés y ligados a las actividades del paciente. Además, es necesario observar y analizar las conversaciones entre el paciente-cuidador y paciente-familiares. Esto podría ofrecer información sobre cuáles son las pautas de los intercambios comunicativos en el contexto familiar, para posteriormente 
brindar orientaciones como parte de la cooperación de los familiares en el proceso de terapia.

Este marco teórico y metodológico genera enormes posibilidades en cuanto a la comprensión de la comunicación con los sujetos afectados de demencia, pero presenta también limitaciones que deben ser manejadas por los profesionales involucrados, como por ejemplo: dedicar más tiempo a la colecta de datos, el no contar con protocolos normatizados y la necesidad de el entrar en lo privado de las relaciones familiares. Consideramos necesario informar de manera clara y suficiente a todas las personas que serán evaluadas, siguiendo las normas básicas de la investigación cualitativa, para ello se pueden seguir recomendaciones para desarrollar investigaciones que involucren conocer las relaciones íntimas de los sujetos evaluados y realizar todos los procedimientos siguiendo un estricto protocolo emanado del comité de bioética correspondiente ${ }^{(31)}$.

Otra limitación a considerar es la ausencia de suficientes datos acerca del rendimiento lingüístico del anciano sano, y sobre el conocimiento aún limitado acerca de las características lingüísticas y su variabilidad interindividual en los diferentes tipos de demencia como por ejemplo en la enfermedad de Huntington, Alzheimer, Parkinson, la demencia Frontotemporal y la enfermedad de los Cuerpos de Lewis, así como la producida por lesiones vasculares, inflamatorias e infecciosas. La investigación actual de los cambios en la expresión verbal de sujetos portadores de alteraciones genéticas relacionadas con la enfermedad de Alzheimer en la fase preclínica ${ }^{(32)}$ pueden ayudar a comprender mejor cómo el intercambio lingüístico en la PCD se va alterando desde momentos tempranos y puede facilitar la comprensión de cómo estos cambios afectan a su interlocutor, quien deberá producir diferentes estrategias buscando ajustarse a estas variaciones.

\section{CONCLUSIONES}

Podemos concluir que estudiar los trastornos del lenguaje desde una perspectiva biopsicosocial en las PCD, remite necesariamente a lo social y a lo cultural como factores fundamentales en la creación de los actos comunicativos, en lo relacionado con los estilos de conversación y con el valor que la demencia tiene en nuestra cultura. Esto lleva a reconsiderar tanto los aspectos teóricos como los metodológicos en el proceso de evaluación lingüística, como vía para desarrollar una actividad más ética con estos pacientes y poder definir una mejor atención del colectivo de PCD y especialmente de contar con mejores recursos para orientar a sus familiares y amigos en lo relacionado con la comunicación.

Es necesario señalar la necesidad de incorporar el análisis discursivo al proceso diagnóstico en Fonoaudiología y estudiar el beneficio de incorporar el estudio del uso de las reparaciones conversacionales por parte de las PCD y sus interlocutores, como un elemento importante en la evaluación y en el tratamiento de esta población. Esto puede ser un excelente recurso para analizar las diferencias discursivas entre los distintos tipos de demencias; especialmente en aquellos tipos de aparición temprana, como es el caso de la demencia Frontotemporal. Al respecto es necesario considerar la necesidad de recursos de evaluación dirigidos a establecer un diagnóstico diferencial, elemento necesario para realizar detecciones tempranas e iniciar un tratamiento de los síntomas lo antes posible. Para alcanzar este objetivo se hace necesario conocer a profundidad el proceso de envejecimiento normal de la población en lo cognoscitivo y en lo lingüístico, como un paso previo, de manera de poder tener un índice normativo de referencia.

Es necesario realizar una revisión ética de los fundamentos de la atención del adulto mayor pues numerosas dudas e interrogantes aun se hacen presentes es este campo, especialmente en torno a la evaluación lingüística del adulto mayor afectado de demencia. Estas limitaciones se deben en nuestra opinión, a la carga negativa que los estereotipos y prejuicios imponen en la percepción de la competencia lingüística del adulto mayor lo que hace complejo definir la norma lingüística en esta población, y en consecuencia, caracterizar con precisión "los trastornos del lenguaje" presentes en el proceso de envejecimiento patológico, y además, al uso exclusivo de herramientas diagnósticas que son importantes pues aportan información necesaria para la planificación de la atención del paciente, pero que no nos ofrecen suficientes datos sobre los intercambios comunicativos que las personas ponemos en marcha en los contextos diarios de vida. Por esto, consideramos necesario integrar al proceso diagnóstico el uso del análisis del discurso de manera de poder valorar el lenguaje como lo que en realidad es: un proceso complejo, dinámico y dependiente de variables lingüísticas y socioculturales.

\begin{abstract}
The purpose of the present study is to reflect on the challenges, perspectives and ethical dilemmas that are present when we assess language of people with dementia. This thought followed two perspectives: an epistemological and ontological one, addressed to study the concept of language disorder and its relation with aging. The other perspective, methodological, related to the analysis of the linguistic assessment techniques used in the diagnosis of the linguistic deficits of this population. Based on these perspectives, we propose useful alternatives, such as to review the concept of disorder, to redefine the objective of language evaluation and to deepen the knowledge of the linguistic functioning of healthy elderly people as a previous step for the use of the discourse analysis as a valid tool to evaluate the linguistic efficiency and to plan the intervention for people with dementia. These actions might guarantee an attention both ethic and adjusted to the actual linguistic needs of this population and their families.
\end{abstract}

Keywords: Alzheimer Disease; Evaluation/methods; Language; Bioethics 


\section{REFERENCIAS}

1. Organización Mundial de la Salud. Promoción de la Salud Mental. Informe Compendiado. Ginebra: Organización Mundial de la Salud; 2004. p.42.

2. Levkoff SE, MacArthur IW, Bucknall J. Elderly mental health in the developing world. Soc Sci Med. 1995;41(7):983-1003.

3. Sinclair AJ. The future of geriatrics - a new horizon or a false dawn? J Nutr Health Aging. 2006;10(4):242-4; discussion 245. Comment in: J Nutr Health Aging. 2006;10(5):431.

4. García Férez J. Bioética y personas mayores. Madri: Informes Portal Mayores. 2003; n4 . [cited 2006 Jun 15]. Available from: http://www. insersomayores.csic.es/documentos/documentos/garcia-bioetica-01. pdf.

5. Bäckman L, Jones S, Berger AK, Laukka EJ, Small BJ. Multiple cognitive deficits during the transition to Alzheimer's disease. J Intern Med. 2004; 256(3):195-204.

6. Moya J. Psicosis única y trastornos del lenguaje. Rev AEN. 1998;(66) Abril/Junio.

7. Pietrosemoli L. Análisis del discurso en poblaciones especiales. En: Bolivar A, editor. Análisis del discurso. Caracas: Universidad Central de Venezuela; 2005. p. 115.

8. Coupland N, Coupland J, Giles H. Language, society and the elderly. Oxford: Basil Blackwell; 1991. p. 14 -5.

9. Tubero AL. A linguagem do envelhecer: saúde e doença. Disturb Comun. 1999;10(2):167-76.

10. Petersen RC. Mild cognitive impairment as a diagnostic entity. J Intern Med. 2004;256(3):183-94.

11. Hamilton HE. Consideraciones éticas sobre la aplicación de la lingüística en contextos clínicos: el caso de la patología del habla-lenguaje. Lengua y Habla. 1996;1(1):51-65.

12. Moreno A, Montañés P, Cano C, Plata S, Gámez A. Comparación de perfiles neuropsicológicos del Viejo-Joven (VJ) y el Viejo-Viejo VV: envejecimiento normal y enfermedad de Alzheimer. Rev Asoc Colomb Gerontol Geriatr. 2005;19(2):776-96.

13. Organización Mundial de la Salud. Clasificación Internacional del Funcionamiento, de la Discapacidad y de la Salud - CIF. Madrid: IMSERSO; 2001. p. 22,142.

14. Hamilton HE. Conversations with an Alzheimer's patient. An interactional sociolinguistic study. Cambridge: Cambridge University Press; 1994. p. 8.

15. Sabat SR. The experience of Alzheimer's disease. Life through a tangled veil. Oxford: Blackwell Publishers; 2001. p. 61.

16. Ripich DN, Terrell BY. Patterns of discourse cohesion and coherence in Alzheimer's disease. J Speech Hear Disord.1988;53(1):8-15.

17. Orange JB, Lubinski RB, Higginbotham DJ. Conversational repair by individuals with dementia of the Alzheimer's type. J Speech Hear Res. 1996;39(4):881-95.

18. Robles Bayón A, Vilariño I. Trastornos del lenguaje en la demencia. En: Juncos Rabadán O. Lenguaje y envejecimiento: bases para la intervención. Barcelona: Masson; 1998. cap. 4, p. 74.
19. Cano Gutiérrez CA, Ramírez Castro RA. Avances nosológicos de las demencias. Caracterización de los pacientes con demencia frontotemporal. Med UNAB. 2004;7(20):84-8.

20. Brauner D, Merel SE. How a model based on linguistic theory can improve the assessment of decision-making capacity for persons with dementia. J Clin Ethics. 2006;17(2):139-48. Comment in: J Clin Ethics. 2006;17(2):99-109.

21. Brauner DJ, Muir JC, Sachs GA. Treating nondementia illnesses in patients with dementia. JAMA. 2000;283(24):3230-5.

22. Patel V, Prince M. Ageing and mental health in developing country: who cares? Qualitative studies from Goa, India. Psychol Med. 2001;31(1):29-38.

23. World Health Organization. Mental Health Determinants and Populations. Department of Mental Health and Substance Dependence. Women's mental health. An evidence based review. Geneva: World Health Organization; 2000.

24. Tchantchou F, Graves M, Ortiz D, Chan A, Rogers E, Shea TB. S-adenosyl methionine: A connection between nutritional and genetic risk factors for neurodegenaration in Alzheimer's disease. J Nutr Health Aging. 2006;10(6):541-4.

25. Folstein MF, Folstein SE, McHugh PR. "Mini-mental state". A practical method for grading the cognitive state of patients for the clinician. J Psychiatr Res. 1975;12(3):189-98.

26. Jorm AF, Christensen H, Jacomb PA, Korten AE, Mackinnon AJ. The cognitive decline scale of the psychogeriatric assessment scales (PAS): longitudinal data on its validity. Int J Geriatr Psychiatry. 2001;16(3):261-5.

27. Roth M, Tym E, Mountjoy CQ, Huppert FA, Hendrie H, Verma S, Goddard R. CAMDEX. A standardised instrument for the diagnosis of mental disorder in the elderly with special reference to the early detection of dementia. Br J Psychiatry. 1986;149:698-709.

28. Valles González B. La cohesión y la coherencia en la conversación del paciente con demencia: un estudio descriptivo. Rev Asoc Colomb Gerontol Geriatr. 2006;20(1):881-7.

29. Pietrosemoli L, Vera M, González Valera S, Coutín P. Marcadores discursivos en hablantes sanos y afásicos: el caso especial de $y$. Bol Lingüística. 2005;17(24):76-91.

30. Schegloff EA, Jefferson G, Sacks H. The preference for selfcorrection in the organization of repair in conversation. Language. 1977;53(2):361-82.

31. Forbat L, Henderson J. "Stuck in the middle with you": the ethics and process of qualitative research with two people in an intimate relationship. Qual Health Res. 2003;13(10):1453-62.

32. Cuetos F, Arango-Lasprilla JC, Uribe C, Valencia C, Lopera F. Linguistic changes in verbal expression: a preclinical marker of Alzheimer's disease. J Int Neuropsychol Soc. 2007;13(3):433-9. 